\title{
Solvation Enthalpies and Gibbs Energies of the Proton and Electron - Influence of Solvation Models
}

\author{
J. Tošović ${ }^{1, *}$, S. Marković1 ${ }^{\text {, D. Milenković }}{ }^{2}$, Z. Marković ${ }^{3}$ \\ ${ }^{1}$ Faculty of Science, University of Kragujevac, 34000 Kragujevac, Serbia \\ e-mail: jelena.tosovic@kg.ac.rs \\ e-mail: mark@kg.ac.rs \\ ${ }^{2}$ Bioengineering Research and Development Center BioIRC, 34000 Kragujevac, Serbia \\ e-mail: deki82@kg.ac.rs \\ ${ }^{3}$ State University of Novi Pazar, 36300 Novi Pazar, Serbia \\ e-mail: zmarkovic@np.ac.rs \\ *corresponding author
}

\begin{abstract}
A comprehensive examination of the solvation enthalpies and Gibbs energies of the proton and electron in twenty solvents of different polarities was carried. Eleven quantum mechanical methods were applied in conjunction with the 6-311++G(d,p) basis set and C-PCM solvation model.

It was found that different methods produce consistent values for the solvation enthalpy and Gibbs energy of the proton in all solvents, while the corresponding values for the electron are mutually notably different. The fact that the Minnesota functionals often produced inconsistent solvation enthalpy and Gibbs energy values of the electron indicates their unreliable performance without the corresponding SMD solvation model, whereas other studied methods only slightly depend on solvation models.

A comparison of the results of the present investigation to those obtained by employing SMD reveals that C-PCM produces slightly more negative (less positive) solvation enthalpies and Gibbs energies of the electron, and less negative values for the proton. These results are also in accord with the literature data where the IEF-PCM solvation model was used.
\end{abstract}

Keywords: Solvated proton, solvated electron, twenty solvents, C-PCM solvation model

\section{Introduction}

Information about the effects of solvent on ion structure and, conversely, how ions influence solvent organization is fundamental to understanding the molecular structure and stability of ions in solution. Ion-solvent interactions are important to the structural, thermodynamic, and dynamic characteristics of many chemical, biological, and atmospheric processes, such as charge transfer reactions, antioxidative processes, catalysis, radiolysis, ion nucleation events, etc. 
The proton and electron are the fundamental charged species. The solvation enthalpy and Gibbs energy of the proton and electron are very important properties that characterize the stability and reactivity of these solvated species. The solvation enthalpy $\Delta H_{\text {sol }}\left(\mathrm{H}^{+}\right)$or Gibbs energy $\Delta G_{\text {sol }}\left(\mathrm{H}^{+}\right)$of the proton is the enthalpy (Gibbs energy) difference between a solvated proton and the proton at rest under vacuum. Accordingly, the solvation enthalpy $\Delta H_{\mathrm{sol}}\left(\mathrm{e}^{-}\right)$or Gibbs energy $\Delta G_{\text {sol }}\left(\mathrm{e}^{-}\right)$of the electron is the enthalpy (Gibbs energy) difference between a solvated electron and the electron at rest under vacuum. The enthalpy and Gibbs energy values of the solvated proton and electron can be calculated from the corresponding solvation enthalpies and Gibbs energies.

Much work has been devoted to achieving accurate hydration enthalpies of the proton (Halliwell and Nyburg 1963; Conway 1964; Marcus 1987; Rashin and Namboodiri 1987; Atkins 1998; Bockris and Reddy 1998; Tissandier et al. 1998; Mejías and Lago 2000; Coe 2001) and electron (Han and Bartels 1990; Schwarz 1991; Shiraishi et al. 1994; Donald et al. 2010). A few results for the hydration Gibbs energies of the proton (Tissandier et al. 1998; Coe 2001) and electron (Han and Bartels 1990) are also available in literature.

On the other hand, the results for solvation enthalpies and Gibbs energies of the proton and electron in other solvents are still limited (Fifen et al. 2011; Fifen et al. 2013; Rottmannová et al. 2013; Marković et al. 2013; Škorňa et al. 2014). In a recent paper, a comprehensive examination of the solvation enthalpies and Gibbs energies of the proton and electron in twenty solvents of different polarities was carried out (Marković et al. 2016). Eleven methods ( $a b$ initio and density functionals) were applied in conjunction with the $6-311++G(d, p)$ basis set and SMD solvation model. The enthalpy and Gibbs energy values for the solvated proton and electron, obtained at the B3LYP/Aug-cc-pVTZ level of theory, were recommended for application in the examinations of antioxidative activity in different solvents.

The aim of this paper is to examine the solvation enthalpies and Gibbs energies of the proton and electron using the same theoretical models in combination with the C-PCM solvation model. The obtained data will allow one to compare and discuss the results from the two sets of calculations.

\section{Methodology}

Our concept is based on the following assumptions: Each molecule of a certain solvent is solvated by the molecules of the same kind (Ssol). When a proton or electron is surrounded by the solvent molecules, it will bind to Ssol, thus yielding a charged particle (S-H) ${ }^{+}$sol or (S-e $)^{-}$sol, which is embedded in dielectric continuum (Fifen et al. 2011; Rottmannová et al. 2013; Marković et al. 2013; Škorňa et al. 2014):

$$
\begin{aligned}
& \mathrm{H}^{+}{ }_{\text {gas }}+\mathrm{S}_{\text {sol }} \rightarrow(\mathrm{S}-\mathrm{H})^{+}{ }_{\text {sol }} \\
& \mathrm{e}^{-} \text {gas } \\
& +\mathrm{S}_{\text {sol }} \rightarrow(\mathrm{S}-\mathrm{e})^{-}{ }_{\text {sol }}
\end{aligned}
$$

Then, the solvation enthalpy $\Delta \mathrm{Hsol}\left(\mathrm{H}^{+}\right)$and Gibbs energy $\Delta \mathrm{Gsol}(\mathrm{H}+)$ of the proton can be calculated as follows:

$$
\begin{aligned}
\Delta H_{\mathrm{sol}}\left(\mathrm{H}^{+}\right) & =H(\mathrm{~S}-\mathrm{H})^{+}{ }_{\text {sol }}-H\left(\mathrm{~S}_{\mathrm{sol}}\right)-H\left(\mathrm{H}^{+}{ }_{\text {gas }}\right) \\
\Delta G_{\mathrm{sol}}\left(\mathrm{H}^{+}\right) & =G(\mathrm{~S}-\mathrm{H})^{+}{ }_{\text {sol }}-G\left(\mathrm{~S}_{\mathrm{sol}}\right)-G\left(\mathrm{H}^{+}{ }_{\text {gas }}\right)
\end{aligned}
$$

The solvation enthalpy $\Delta \operatorname{Hsol}\left(\mathrm{e}^{-}\right)$and Gibbs energy $\Delta \mathrm{Gsol}\left(\mathrm{e}^{-}\right)$of the electron are defined analogously: 


$$
\begin{aligned}
& \Delta H_{\text {sol }}\left(\mathrm{e}^{-}\right)=H(\mathrm{~S}-\mathrm{e})^{-\bullet}{ }_{\text {sol }}-H\left(\mathrm{~S}_{\text {sol }}\right)-H\left(\mathrm{e}^{-}{ }_{\text {gas }}\right) \\
& \Delta G_{\text {sol }}\left(\mathrm{e}^{-}\right)=G(\mathrm{~S}-\mathrm{e})^{-\bullet}{ }_{\text {sol }}-G\left(\mathrm{~S}_{\text {sol }}\right)-G\left(\mathrm{e}^{-}{ }_{\text {gas }}\right)
\end{aligned}
$$

The equations (1) - (6) were used to compute the solvation enthalpies and Gibbs energies of the proton and electron. For this purpose the following quantum mechanical methods were applied: MP2, B2PLYP, B2PLYP-D3, B3LYP, B3LYP-D2, B3LYP-D3, M05-2X, M06, M062X, M06-HF, and TPSS. The calculations were performed for the following solvents: 1-butanol (dielectric constant $\varepsilon=17.33)$, acetic acid $(\varepsilon=6.25)$, acetone $(\varepsilon=20.49)$, acetonitrile $(\varepsilon=$ $35.69)$, aniline $(\varepsilon=6.89)$, benzaldehyde $(\varepsilon=18.22)$, benzene $(\varepsilon=2.27)$, chlorobenzene $(\varepsilon=$ 5.70), cyclohexane $(\varepsilon=2.02)$, diethylether $(\varepsilon=4.24)$, dimethylformamide (DMF, $\varepsilon=37.22)$, dimethyl sulfoxide (DMSO, $\varepsilon=46.83)$, ethanol $(\varepsilon=24.85)$, methanol $(\varepsilon=32.61)$, nitrobenzene $(\varepsilon=34.81)$, pentylethanoate $(\varepsilon=4.73)$, quinoline $(\varepsilon=9.16)$, tetrahydrofuran (THF, $\varepsilon=7.43)$, toluene $(\varepsilon=2.37)$, and water $(\varepsilon=78.35)$.

\subsection{Computational details}

The Gaussian 09 program package was employed for all calculations within this paper (Frisch et al. 2013). All applied methods were combined with the split-valence triple-zeta 6$311++G(d, p)$ basis set. The geometries of all parent solvent molecules and corresponding protonated molecules and radical anions were fully optimized, and frequency calculations were carried out. For this purpose the conductor-like polarizable continuum solvation model (CPCM) was applied (Cossi et al. 2003). An analysis of the results of the frequency calculations showed that there were no imaginary vibrations, implying that all revealed stationary points are equilibrium geometries. The enthalpy and Gibbs energy values were calculated at $\mathrm{P}=101325$ $\mathrm{Pa}$ and $\mathrm{T}=298.15 \mathrm{~K}$.

Note that exactly the same quantum mechanical methods and basis set were used in the present work and in the recent related study (Marković et al. 2016). Such approach will enable a comparison between the performances of the two solvation models: SMD and C-PCM.

\subsection{Survey of the applied quantum mechanical methods}

In MP2 (Head-Gordon et al. 1988), the Hartre-Fock method is corrected by means of the second-order perturbation theory, thus introducing the effects of electron correlation.

Hybrid exchange-correlation functionals are constructed as linear combinations of the exact exchange functional, as defined within the Hartree-Fock theory, and the exchange and correlation functionals, as defined within the DFT theory. The hybrid exchange-correlation energy can be presented by equation (7):

$$
E_{\mathrm{XC}}^{\text {hibrid }}=\mathrm{c}_{\mathrm{HF}} E_{\mathrm{X}}^{\mathrm{HF}}+\mathrm{c}_{\mathrm{DFT}} E_{\mathrm{XC}}^{\mathrm{DFT}}
$$

where $E_{\mathrm{X}}^{\mathrm{HF}}$ and $E_{\mathrm{XC}}^{\mathrm{DFT}}$ stand for the non-local Hartree-Fock exchange energy, and local DFT exchange-correlation energy, whereas $\mathrm{C}_{\mathrm{HF}}$ and $\mathrm{C}_{\mathrm{DFT}}$ are parameters. A widely used B3LYP method (Becke 1993) combines the Hartree-Fock exchange functional with the exchange functionals $E_{\mathrm{X}}^{\mathrm{LDA}}$ and $E_{\mathrm{X}}^{\mathrm{B} 88}$, correlation functionals $E_{\mathrm{C}}^{\mathrm{LYP}}$ and $E_{\mathrm{C}}^{\mathrm{VWN}}$, and exchangecorrelation functional $E_{\mathrm{XC}}^{\mathrm{LDA}}$.

Grimme proposed the B2PLYP method (Grimme 2006a), where the expression for the exchange-correlation energy $\mathrm{E}_{\mathrm{xc}}$ is given as follows:

$$
E_{\mathrm{XC}}^{\mathrm{B} 2 \mathrm{PLYP}}=E_{\mathrm{XC}}^{\mathrm{B} 2 \mathrm{LYP}}+\left(1-a_{\mathrm{C}}\right) E_{\mathrm{C}}^{\mathrm{MP} 2}
$$


where the exchange-correlation energy from the B2PLYP functional is combined with the MP2 correlation energy which is scaled by $\left(1-\mathrm{a}_{\mathrm{C}}\right)$. $\mathrm{a}_{\mathrm{C}}$ is a parameter determined by a parameterization where the heats of formation of the G2/97 set (Curtiss et al. 2000) were used.

The DFT-D functionals of Grimme contain additional terms in the formulae for the total energy, whose purpose is to take into account dispersion forces:

$$
E_{\mathrm{DFT}-\mathrm{D}}=E_{\mathrm{DFT}}+E_{\mathrm{disp}}
$$

Thus, dispersion-corrected methods are not new methods. Actually, B3LYP-D2, B3LYPD3, B2PLYP-D2, and B2PLYP-D3 denote calculations with the usual B3LYP and B2PLYP methods, plus a D2 or D3 dispersion correction energy terms. These energy terms are functions of interatomic distances. Adjustable parameters of these functions are fitted to the energies computed using the CCSD(T)/CBS model. The DFT-D2 energy correction considers all pairs of atoms, whereas DFT-D3 correction considers all triplets of atoms (Grimme 2006b; Grimme et al. 2010).

In the theory of the hybrid meta-GGA functionals the hybrid energy is given by the formula:

$$
E_{\mathrm{XC}}^{\text {hybrid }}=\frac{\mathrm{X}}{100} E_{\mathrm{X}}^{\mathrm{HF}}+\left(1-\frac{\mathrm{X}}{100}\right) E_{\mathrm{X}}^{\mathrm{DFT}}+E_{\mathrm{C}}^{\mathrm{DFT}}
$$

where $\mathrm{X}$ represents the percentage of the Hartree-Fock exchange in the hybrid functional, and $E_{\mathrm{X}}^{\mathrm{DFT}}$ and $E_{\mathrm{C}}^{\mathrm{DFT}}$ are the local DFT exchange and correlation energies. M05-2X (Zhao et al. 2006), M06, M06-2X, and M06-HF (Zhao and Truhlar 2008) are global hybrid functionals with 52, 27, 54, and $100 \%$ Hartree-Fock exchange, respectively. TPSS is a meta-GGA nonempirical exchange-correlation functional (Tao et al. 2003).

\section{Results and discussion}

Following the above described procedure, the solvation enthalpies and Gibbs energies of the proton and electron in twenty commonly used solvents are determined. As for $\mathrm{H}_{\left(\mathrm{H}^{+}\right.}$gas $)$, $H\left(\mathrm{e}^{-}\right.$gas $), G\left(\mathrm{H}^{+}\right.$gas $)$, and $G\left(\mathrm{e}^{-}\right.$gas $)$, the commonly accepted values were used: $6.197 \mathrm{~kJ} \mathrm{~mol}^{-1}$ for the proton enthalpy, $3.146 \mathrm{~kJ} \mathrm{~mol}^{-1}$ for the electron enthalpy, $-26.255 \mathrm{~kJ} \mathrm{~mol}^{-1}$ for the proton Gibbs energy, and $-3.633 \mathrm{~kJ} \mathrm{~mol}^{-1}$ for the electron Gibbs energy (Bartmess 1994). The results of our calculations are illustrated with Fig. 1, and summarized in Tables $1-4$.

The electrostatic potential maps, as well as the partial charges, of solvated methanol and corresponding charged species satisfy one's expectations. The red and blue regions in the electrostatic potential map of methanol correspond to the polarized O-H group. The surfaces for the protonated methanol and radical anion are almost fully coloured blue and red, thus revealing that the positive and negative charge are delocalized over the entire species. In the radical anion the hydroxyl hydrogen bears appreciable large negative charge of -0.554 . This finding supports our selection of the basis set that includes the diffuse functions on the hydrogens. 


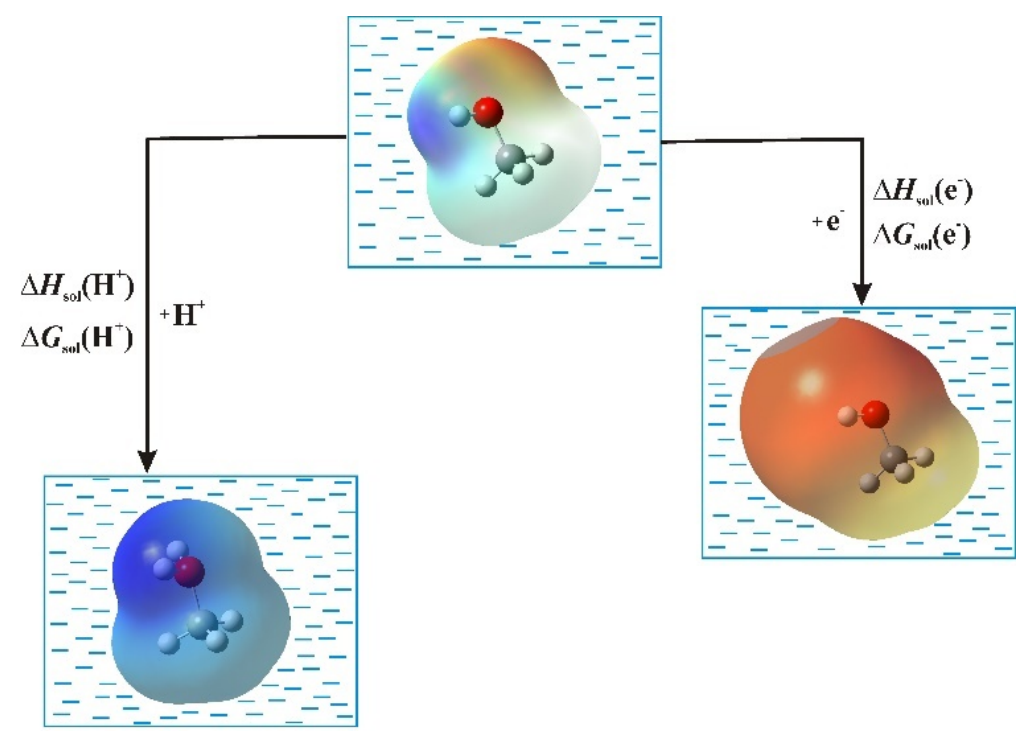

Fig. 1. Electrostatic potential maps of solvated methanol molecule (top), its radical anion (left), and protonated methanol (right)

\begin{tabular}{|c|c|c|c|c|c|c|c|c|c|c|c|c|}
\hline Solventsa & MP2a & B2PLYPa & B2PLYP-D3a & B3LYPa & B3LYP-D2a & B3LYP-D3a & M05-2Xa & M06a & M06-2Xa & M06-HFa & TPSSa & RSDáa \\
\hline 1-Butanola & -1014.10 & -1013.5 & $-1014.9 \square$ & -1014.10 & $-1017.6 \square$ & $-1017.2 \square$ & $-1010.0 \mathrm{a}$ & $-1006.8 \mathrm{a}$ & $-1008.4 a$ & $-1016.2 a$ & $-1022.5 \mathrm{a}$ & $\overline{0.42 \mathrm{a} a}$ \\
\hline Acet & & & & & & & & 8a & & & $6 \mathrm{a}$ & $0.35 \mathrm{ad}$ \\
\hline Acetone & $-1021.4 a$ & $-1026.6 \square$ & -1027.7 a & $-1031.1 a$ & & $-1033.8 \square$ & $-1026.5 \square$ & $-1029.5 a$ & $-1024.3 a$ & $-1020.5 \square$ & $-1037.7 a$ & 0.50 마 \\
\hline Acetonitrile & 970 & $-1003,4 \mathrm{q}$ & $-1004.2 a$ & -1009.1 a & 0080 & 101090 & 905 9r & $-1011.0 \mathrm{a}$ & $997,2 \pi$ & -990.0 व & -1016.10 & 0.76 a d \\
\hline & $0.4 \mathrm{r}$ & & & & & & & & & & & $0.54 a d a$ \\
\hline g. & & & & & & & & & & & & 0.82 마 \\
\hline B & & -8 & & & & & & & & & & oda \\
\hline zened & & & & & & & & & & & & $19 \mathrm{ada}$ \\
\hline & & & & & & & & & & & & 1.11 a d \\
\hline & & & & & & & & & & & & $6 \mathrm{ad}$ \\
\hline & & & & & & & & & & & & 0.39 a d \\
\hline & & & & & & & & & & & & $0.28 a$ a \\
\hline & $-1019.3 a$ & $-1018.1 a$ & & & & & & & -10 & -1019.1 a & $5.9 \mathrm{a}$ & $0.37 \mathrm{ar}$ \\
\hline Meth & $-1018.2 a$ & -1014.5 口а & -1015.5 a & -1013.1 a & 5.4 व & -1015.2 마 & $-1011.3 \square$ & -1008.7 ㅁ & $-1010.5 a$ & -1018.3 ㅁ & $-1020.2 \square$ & 0.33 a d \\
\hline Nitrobenzen & $-980.5 a$ & $-986.3 a$ & $-987.3 a$ & $-992.7 a$ & $-994.8 \square$ & -995.1 a & $-973.8 a$ & -986.0 ฉ & $-978.4 \mathrm{a}$ & $-969.8 a$ & $-1004.2 a$ & $0.98 \mathrm{ad}$ \\
\hline Pentylethanoate & -989.4 ㅁ & -993.10 & $2 \mathrm{r}$ & $9968 \mathrm{r}$ & 8 & & $-990.2 a$ & -990.3 口а & & 30 & $-1003.2 a$ & $0.49 \mathrm{ad}$ \\
\hline & 10 & & & & & & & & & 10 & .10 & 0.79 마 \\
\hline THI & 02000 & $6 a$ & & a & & $4 \mathrm{a}$ & $5 \mathrm{a}$ & 6a & .00 & $.2 \mathrm{\alpha}$ & $.9 \mathrm{a}$ & $0.47 \mathrm{ara}$ \\
\hline & & & & & & & & & & & & 2.15 a d \\
\hline Watera & -997.3 口 & $-992.0 \mathrm{a}$ & -992.5 며 & -990.7 a & -991.3 미 & -992.0 व & $-988.7 a$ & -993.0 미 & -991.9 미 & $-992.5 \mathrm{a}$ & -998.8 a & $0.28 \mathrm{ad}$ \\
\hline
\end{tabular}

Table 1. Solvation enthalpies of the proton $\left(\mathrm{kJ} \mathrm{mol}^{-1}\right)$ in the studied solvents. RSD (\%) denotes the relative standard deviation 


\begin{tabular}{|c|c|c|c|c|c|c|c|c|c|c|c|c|}
\hline Solventsa & MP2a & B2PLYPa & B2PLYP-D3a & B3LYPa & B3LYP-D2a & B3LYP-D3a & M05-2Xa & M06a & M06-2Xa & M06-HFa & TPSSa & RSDa a \\
\hline 1-Butanola & -1013.5 a & -1014.5 a & $-1015.7 \mathrm{a}$ & $-1015.8 \mathrm{a}$ & $-1018.8 \propto$ & $-1018.6 a$ & $-1011.4 a$ & $-1008.1 a$ & $-1009.4 \alpha$ & $-1017.6 a$ & $-1026.7 \alpha$ & $0.48 \mathrm{a} a$ \\
\hline Acetic acida & -978.5 미 & $-981.3 a$ & $-982.6 a$ & $-982.5 \square$ & -987.4 口 & -987.4口 & $-979.6 a$ & -984.6 a & -983.4 a & $-979.3 a$ & -987.6 a & $0.32 \mathrm{ad}$ \\
\hline Acetoned & $-1021.8 \mathrm{a}$ & $-1027.3 a$ & $-1028.5 a$ & $-1031.9 a$ & $-1034.2 \mathrm{a}$ & -1034.5 a & $-1026.5 a$ & $-1030.1 a$ & $-1024.7 a$ & $-1019.4 a$ & $-1039.1 a$ & $0.54 a \mathrm{a}$ \\
\hline Acetonitriled & $-999.2 \propto$ & $-1004.8 \mathrm{a}$ & $-1005.6 \mathrm{a}$ & $-1013.3 a$ & -1014.0 a & $-1015.1 a$ & $-1000.0 a$ & $-1015.1 \mathrm{a}$ & $-1001.2 a$ & -994.00 & $-1020.5 a$ & $0.80 \mathrm{ar}$ \\
\hline Anilinea & $-1082.6 a$ & -1075.5 미 & $-1077.2 a$ & $-1075.0 a$ & $-1078.0 \mathrm{a}$ & $-1079.0 \mathrm{a}$ & $-1067.9 a$ & $-1064.2 a$ & $-1066.8 a$ & -1067.4 a & $-1081.8 a$ & $0.57 \mathrm{ad}$ \\
\hline Benzaldehydea & $-1013.6 a$ & -1024.6 a & $-1025.6 a$ & $-1031.3 a$ & -1033.5 a & $-1033.6 a$ & $-1020.7 a$ & $-1024.5 a$ & $-1016.9 a$ & -1012.0 a & $-1038.7 a$ & $0.81 \mathrm{ag}$ \\
\hline Benzenea & $-855.9 a$ & $-876.0 a$ & $-875.4 a$ & $-886.4 a$ & $-890.8 a$ & -889.0 a & $-862.2 a$ & $-884.8 a$ & $-861.3 a$ & $-835.1 a$ & $-896.7 a$ & $2.02 \mathrm{ad}$ \\
\hline Chlorobenzened & -847.9 a & $-847.2 a$ & -848.8 a & $-850.4 \square$ & -852.9 ㅁ & -853.7 ㅁ & -833.1 a & -846.0 ㅁ & $-831.2 a$ & -823.0 व & $-861.8 a$ & $1.28 \mathrm{ad}$ \\
\hline Cyclohexaned & $-761.7 a$ & $-741.8 \propto$ & $-743.8 \propto$ & $-749.6 a$ & -754.0 a & $-753.9 \mathrm{a}$ & $-741.3 a$ & $-742.4 a$ & $-736.8 \mathrm{a}$ & $-741.8 \propto$ & $-764.9 a$ & $1.17 \mathrm{ar}$ \\
\hline Diethylethera & $-995.3 \alpha$ & $-998.1 a$ & $-1000.7 a$ & $-999.1 \alpha$ & $-1003.6 a$ & $-1004.9 a$ & $-995.8 \propto$ & $-987.3 \mathrm{a}$ & $-991.0 \mathrm{a}$ & $-999.9 \alpha$ & $-1004.5 a$ & $0.53 \mathrm{ad}$ \\
\hline DMFa & -1082.0 a & -1080.5 a & $-1081.8 a$ & -1079.5 ㅁ & -1082.0 a & $-1081.9 a$ & $-1079.4 \square$ & $-1070.3 a$ & -1079.0 a & -1087.0 व & $-1083.3 a$ & $0.36 \mathrm{ad}$ \\
\hline DMSOA & $-1102.1 a$ & $-1098.8 a$ & $-1101.2 \alpha$ & $-1098.8 \mathrm{a}$ & $-1096.6 \mathrm{a}$ & $-1097.8 a$ & $-1096.2 a$ & $-1094.8 a$ & $-1098.0 \propto$ & $-1103.9 a$ & $-1101.4 a$ & $0.24 a x$ \\
\hline Ethanold & -1018.8 & -1018.7 口 & -1020.0 a & -1019.9 a & $-1022.6 a$ & -1022.5 a & -1016.6 & $-1014.2 \mathrm{a}$ & -1015.5 a & -1020.5 a & -1028.9 a & $0.37 \mathrm{ad}$ \\
\hline Methanol $\square$ & -1019.6 & $-1015.9 a$ & $-1016.8 a$ & -1014.8 a & $-1016.8 a$ & $-1016.6 a$ & -1013.1 a & $-1010.3 a$ & $-1012.2 a$ & $-1020.3 a$ & -1021.8 & 0.33 a d \\
\hline Nitrobenzened & $-982.1 a$ & $-986.2 \alpha$ & $-987.2 \alpha$ & $-994.4 a$ & -995.0 व & $-995.4 a$ & $-975.7 a$ & $-988.2 \propto$ & $-980.2 a$ & $-971.1 a$ & $-1006.5 a$ & $0.98 \mathrm{ar}$ \\
\hline Pentylethanoate & -985.0 व & -988.2 व & -988.5 口а & $-993.3 \square$ & -997.4 口 & -994.6 口а & $-990.2 \square$ & $-987.9 \square$ & $-987.2 \square$ & -988.1 a & $-1000.8 a$ & $0.47 \mathrm{ad}$ \\
\hline Quinolinea & /a & -1119.1 a & $120.6 a$ & -1125.10 & $-1129.5 a$ & $-1128.7 a$ & -1112.0 a & $-1115.2 \mathrm{a}$ & $-1108.6 a$ & -1103.6 & $-1130.5 a$ & $0.79 \mathrm{ad}$ \\
\hline THFa & $-1018.1 a$ & -1022.5 a & $-1024.2 a$ & $-1023.9 a$ & -1028.5 a & $-1027.7 \mathrm{a}$ & $-1018.7 \mathrm{a}$ & $-1012.9 a$ & $-1014.8 \mathrm{a}$ & $-1021.3 \mathrm{a}$ & $-1027.6 a$ & $0.49 \mathrm{ada}$ \\
\hline & -877.8 a & $-907.9 a$ & -909.2 व & & -923.2 व & -919.2 미 & -895.1 只 & -921.1 口 & -894.8 a & -868.9 व & -922.2 口а & $1.98 \mathrm{ad}$ \\
\hline Watera & $-1001.2 \mathrm{a}$ & $-996.0 \mathrm{a}$ & $-996.5 \mathrm{a}$ & $-994.7 a$ & -995.3 a & -996.0 व & $-990.0 a$ & $-994.3 \mathrm{a}$ & $-993.2 \alpha$ & $-993.8 a$ & $-1000.0 a$ & $0.29 \mathrm{ad}$ \\
\hline
\end{tabular}

Table 2. Solvation Gibbs energies of the proton $\left(\mathrm{kJ} \mathrm{mol}^{-1}\right)$ in the studied solvents. RSD (\%) denotes the relative standard deviation

\begin{tabular}{|c|c|c|c|c|c|c|c|c|c|c|c|c|}
\hline Solventsa & MP2a & B2PLYPa & B2PLYP-D3a & B3LYPa & B3LYP-D2a & B3LYP-D3a & M05-2Xa & M06a & M06-2Xa & M06-HFa & TPSSa & RSDa $\mathrm{d}$ \\
\hline 1-Butanolda & -30.6 a & $-85.9 a$ & $-85.9 a$ & $-101.0 a$ & -100.8 口 & $-100.9 a$ & -73.5 a & -74.5 a & -84.1 a & $-101.8 \mathrm{a}$ & -95.4 미 & $23.4 \mathrm{a} \mathrm{a}$ \\
\hline Acetic acida & $-60.3 a$ & $-89.9 \mathrm{a}$ & $-89.8 \mathrm{a}$ & $-88.9 a$ & $-89.1 \mathrm{a}$ & -88.8 a & $-97.8 \mathrm{a}$ & $-91.5 a$ & $-91.3 a$ & $-109.3 a$ & $-108.6 \mathrm{a}$ & $13.4 \mathrm{a} \mathrm{a}$ \\
\hline Acetonea & -110.10 & $-133.6 a$ & $-133.5 \mathrm{a}$ & $-154.3 a$ & $-154.5 \mathrm{a}$ & -154.10 & $-142.2 a$ & $-142.0 \mathrm{a}$ & $-137.6 a$ & -151.1 a & $-151.8 \mathrm{a}$ & $9.0 \mathrm{a}$ \\
\hline Acetonitrilea & $-64.2 a$ & $-77.9 \mathrm{a}$ & $-77.9 \alpha$ & $-133.3 a$ & -134.0 a & $-133.4 \alpha$ & $-114.8 \alpha$ & $-114.3 \mathrm{a}$ & $-111.6 a$ & $-120.6 a$ & $-130.0 \alpha$ & $21.9 \mathrm{a}$ \\
\hline Anilined & -48.2 ㅁ & $-48.4 \square$ & $-47.9 a$ & -75.1 a & $-75.2 \square$ & -75.0 ㅁ & $-77.4 \square$ & -83.5 a & -77.9 व & $-91.7 \mathrm{a}$ & -73.7 口 & $20.5 \mathrm{a}$ a \\
\hline Benzaldehyde & -118.6 & $-226.2 a$ & -226.0 마 & $-253.3 a$ & -253.5 & -253.0 व & -252.10 & -251.3 미 & -246.6 & $-255.6 a$ & $-249.8 \square$ & 16.2 a \\
\hline Benzenea & $9.9 a$ & $2.0 \mathrm{a}$ & $3.5 a$ & $-18.2 a$ & -19.10 & $-17.4 a$ & $-11.2 a$ & $-22.6 a$ & $-11.9 a$ & -23.10 & $-17.3 a$ & 95.1 a \\
\hline Chlorobenzened & $-31.1 a$ & $-81.5 a$ & $-81.3 \mathrm{o}$ & $-107.9 a$ & -108.7 a & $-107.4 \alpha$ & $-104.8 \alpha$ & $-110.9 a$ & $-104.7 \mathrm{a}$ & $-112.4 a$ & $/ a$ & $25.10 \mathrm{a}$ \\
\hline Cyclohexanea & $22.4 a$ & $10.0 \mathrm{a}$ & $10.1 a$ & $-7.6 a$ & $-6.9 a$ & $-7.3 a$ & 36.10 & 22.90 & $23.3 a$ & -13.20 & $1.9 a$ & $186.10 \mathrm{a}$ \\
\hline Diethylether $\square$ & -10.6 미 & $-19.4 \square$ & $-19.2 \square$ & $-41.4 \square$ & $-41.6 a$ & $-41.4 \square$ & -5.0 a & $-14.0 \mathrm{a}$ & $-20.4 \square$ & $-50.7 \mathrm{a}$ & -32.0 ㅁ & $53.8 \mathrm{a} \mathrm{a}$ \\
\hline DMFa & -76.1 口 & -94.9 व & $-95.1 \mathrm{a}$ & $-79.8 \square$ & $-80.3 a$ & -79.8 미 & $-106.0 \mathrm{a}$ & -107.3 므 & -107.8 ㅁ & -117.0 a & -108.3 a & 14.5 a d \\
\hline DMSOa & $-5.9 \mathrm{a}$ & -67.10 & -67.10 & $-83.3 a$ & 78 & $-83.4 a$ & -51.10 & $-62.8 a$ & $-65.0 a$ & $-87.0 \propto$ & $-77.9 \mathrm{a}$ & $33.0 \mathrm{ad}$ \\
\hline Eth & $-80.5 a$ & 50 & -89 & $3 a$ & 10 & 20 & 10 & $6 a$ & a & $2 a$ & $-99.5 a$ & $12.5 \mathrm{a}$ a \\
\hline Methanola & $-84.5 a$ & -94.80 & $-94.8 \mathrm{a}$ & $-111.3 a$ & -111.3 a & $-111.3 \mathrm{~d}$ & $-81.9 a$ & $-83.3 a$ & $-93.5 a$ & $4 a$ & $-104.6 a$ & $12.0 \mathrm{ag}$ \\
\hline Nitrol & $-160.9 a$ & $-307.0 a$ & -309.7 口 & $-342.3 a$ & -342.7 a & $-342.3 a$ & -348.8 & $-337.9 \square$ & -341.5 a & -369.0 & $-325.3 \mathrm{a}$ & $16.6 \mathrm{a}$ \\
\hline noated & -41.3 a & $-66.8 \square$ & $-66.6 a$ & $-88.2 a$ & $-88.2 a$ & -87.8 a & -75.7 미 & $-73.1 \mathrm{a}$ & $-72.4 a$ & -86.0 미 & -85.2 미 & $17.9 \mathrm{a}$ a \\
\hline Quinolined & $/ \alpha$ & $-187.5 a$ & $-187.4 a$ & $-208.7 \not$ & -209.0 a & $-208.3 \alpha$ & $-207.8 \alpha$ & $-212.7 \mathrm{a}$ & $-205.3 \mathrm{~d}$ & $-210.5 a$ & $-206.4 \alpha$ & $4.2 \mathrm{a}$ \\
\hline THFo & $-35.6 a$ & $-40.7 \mathrm{a}$ & $-40.6 a$ & $-59.6 a$ & $-59.4 a$ & $-59.4 a$ & $-27.6 a$ & $-37.7 a$ & $-40.5 a$ & $-70.0 \mathrm{a}$ & $-48.4 \mathrm{a}$ & $26.4 \propto$ a \\
\hline Toluened & $/ a$ & $-1.9 \square$ & -1.9 a & $-26.7 a$ & -27.1 a & $-26.3 \square$ & $-16.9 a$ & $-28.7 a$ & $-18.3 \square$ & $-30.4 a$ & $-24.9 \square$ & 49.5 a \\
\hline Waterd & -96.5 미 & $-112.2 a$ & -112.2 口 & $-134.2 a$ & -134.2 a & $-134.2 a$ & $-100.8 a$ & $-99.6 a$ & -109.9 a & $-131.7 a$ & $-121.9 a$ & $12.10 \mathrm{a}$ \\
\hline
\end{tabular}

Table 3. Solvation enthalpies of the electron $\left(\mathrm{kJ} \mathrm{mol}^{-1}\right)$ in the studied solvents. RSD (\%) denotes the relative standard deviation

\begin{tabular}{|c|c|c|c|c|c|c|c|c|c|c|c|c|}
\hline Solventsa & MP2a & B2PLYPa & B2PLYP-D3a & B3LYPa & B3LYP-D2a & B3LYP-D3a & M05-2Xa & M06a & M06-2Xa & M06-HFa & TPSSa & RSDa a \\
\hline 1-Butanola & $-31.9 a$ & $-88.4 a$ & -88.3 a & $-103.2 a$ & $-102.8 a$ & $-102.7 a$ & $-75.3 a$ & -76.5 a & $-85.7 a$ & $-102.5 a$ & -97.5 a & $23.0 \mathrm{ad}$ \\
\hline Acetic acida & $-62.3 a$ & -90.5 a & -90.5 a & $-92.8 \square$ & -92.4 口а & -93.0 ㅁ & $-98.9 a$ & -93.2 口 & -94.0 व & -112.3 a & -107.1 a & $12.8 \mathrm{ad}$ \\
\hline Acetonea & -108.8 & $-132.6 \mathrm{a}$ & -132.5 ㅁ & -153.6 & $-154.7 a$ & $-153.9 \mathrm{a}$ & -142.3 a & -142.40 & -138.1 口 & 51.4 a & $-151.2 \mathrm{a}$ & 9.3 ad \\
\hline Acetonitrilea & $-67.0 \mathrm{a}$ & $-90.9 a$ & $-91.0 \mathrm{a}$ & $-140.5 \mathrm{a}$ & $-140.2 \alpha$ & $-141.3 \mathrm{a}$ & $-122.0 \propto$ & $-121.7 a$ & $-118.8 a$ & $-127.8 \alpha$ & $-137.2 \alpha$ & $20.0 \mathrm{ad}$ \\
\hline Anilinea & $-50.0 a$ & -50.1 da & $-49.7 a$ & -78.10 & -78.0 a & -78.0 a & $-85.6 a$ & -91.10 & $-87.5 a$ & $-97.9 a$ & $-76.3 a$ & 22.0 ad \\
\hline Benzaldehydea & -118.4 & $-229.3 a$ & -229.2 व & $-255.2 \square$ & $.2 a$ & $-255.4 \mathrm{a}$ & -25 & $-253.3 a$ & -248.4 & -257.3 a & -251.9 a & $16.3 a \mathrm{~d}$ \\
\hline Benz & 7.8 a & $-0.7 a$ & & $-26.6 a$ & $2 \mathrm{a}$ & qa & -18 & $-29.3 a$ & $-17.7 \alpha$ & $-27.1 a$ & $-22.2 \mathrm{a}$ & $79.6 \mathrm{ad}$ \\
\hline enzened & $-33.0 \mathrm{a}$ & $2 \mathrm{a}$ & $9 a$ & $-118.0 \mathrm{a}$ & $-118.1 a$ & $-118.9 \mathrm{a}$ & $-115.4 \mathrm{a}$ & $-119.5 \mathrm{a}$ & $-120.9 \mathrm{a}$ & $-119.4 \mathrm{a}$ & $/ \mathrm{a}$ & $25.6 \mathrm{da}$ \\
\hline Cyclo & $18.8 \mathrm{a}$ & $3.7 \alpha$ & & $-13.8 \propto$ & -12.10 & $-11.5 \mathrm{a}$ & $31.2 \alpha$ & $17.8 \mathrm{a}$ & $18.9 \mathrm{a}$ & $-14.8 \alpha$ & $-2.9 a$ & $431.4 \mathrm{ad}$ \\
\hline Dieth & $-12.2 a$ & -19.8 a & $7 a$ & & & & $-6.6 a$ & $-15.3 \mathrm{a}$ & & & $-34.3 \square$ & 51.7 마 \\
\hline DMFa & $-74.2 a$ & -94.1 a & $2 \mathrm{a}$ & 10 & 2ם & 4 口а & -105.1 미 & -106.8 a & -106.6 口 & -116.7 미 & -108.5 며 & 14.2 ad \\
\hline & -9.1 a & & & & & & & & & & & $31.8 \mathrm{ad}$ \\
\hline Ethan & $-82.3 a$ & $-91.5 a$ & $-91.5 a$ & $2 a$ & .10 & $0 \alpha$ & $-78.3 a$ & $-80.5 a$ & $-89.4 \alpha$ & -111.10 & $-101.3 a$ & $12.0 \mathrm{ara}$ \\
\hline Methanola & $-86.9 a$ & $-97.4 a$ & $-97.4 \alpha$ & $-113.9 a$ & .0 a & $-114.0 a$ & $-84.2 \alpha$ & $-86.3 a$ & $-95.5 \alpha$ & $-116.4 a$ & $-107.5 a$ & $11.7 \mathrm{da}$ \\
\hline Nitro & -162.3 미 & $-308.0 \mathrm{a}$ & -308.1 마 & -344.3 미 & $-344.4 a$ & -343.1 व & -351.3 口 & -340.0 a & -343.6 ㅁ & -371.6 & $-327.6 a$ & 16.6마 \\
\hline Pentylethanoated & $-39.2 a$ & $-64.7 \mathrm{a}$ & $-63.9 \mathrm{a}$ & $-86.5 a$ & -90.4 口 & $-88.3 \square$ & $-74.9 a$ & $-72.8 \square$ & $-72.1 \mathrm{a}$ & $-87.6 \mathrm{a}$ & $-84.8 \square$ & $19.4 \square \mathrm{d}$ \\
\hline Quinolined & $/ \mathrm{a}$ & $-193.0 \propto$ & $-192.9 a$ & -212.4 a & $-212.4 \alpha$ & $-212.7 a$ & $-211.3 \mathrm{a}$ & $-216.3 a$ & $-208.9 \mathrm{a}$ & -214.1 a & $-210.1 a$ & $3.8 \mathrm{da}$ \\
\hline THFa & $-35.8 a$ & $-43.0 \mathrm{a}$ & $-42.9 a$ & $-62.0 a$ & $-62.0 a$ & $-62.0 a$ & -31.1 a & $-39.6 a$ & -43.10 & $-71.9 a$ & $-50.1 a$ & $25.3 \mathrm{ad}$ \\
\hline Toluene & $/ a$ & $-4.2 \alpha$ & -5.0 व & -26.5 a & -29.5 & -26.8 & $-19.6 a$ & -32.0 a & $-21.2 a$ & $-32.2 \alpha$ & $-19.9 a$ & 44.2 व व \\
\hline Watera & $-98.4 a$ & $-114.1 a$ & -114.1 미 & $-136.1 \mathrm{a}$ & $-136.1 a$ & -136.10 & -102.8 a & $-101.6 a$ & -111.8 व & $-133.6 a$ & $-125.6 a$ & $11.9 \mathrm{ada}$ \\
\hline
\end{tabular}

Table 4. Solvation Gibbs energies of the electron $\left(\mathrm{kJ} \mathrm{mol}^{-1}\right)$ in the studied solvents. RSD (\%) denotes the relative standard deviation 
It is worth mentioning that we experienced very similar problems to those that have appeared when the SMD solvation model has been used (Marković et al. 2016). As a consequence of convergence failure and undesired imaginary vibrations we were not able to determine the solvation enthalpy and Gibbs energy values for the electron in quinolone and toluene using the MP2 method, and in chlorobenzene using the TPSS functional (Tables 3 and 4). Also, the MP2 method failed to produce the solvation enthalpy and Gibbs energy of the proton in quinolone (Tables 1 and 2). In addition, all methods in combination with the C-PCM solvation model failed to optimize protonated chloroform and carbon tetrachloride, and yielded the structures consisting of separated $\mathrm{HCl}$ and corresponding cation. The same undesired result has been obtained in combination with the SMD solvation model.

At first glance, the results of the present investigation are very similar to those reported recently (Marković et al. 2016). On one hand, different methods produce consistent values for the solvation enthalpy and Gibbs energy of the proton in all solvents, where the RSD (relative standard deviation) values go beyond $2 \%$ only in the case of benzene and toluene (Tables 1 and 2). On the other hand, the solvation enthalpy and Gibbs energy values for the electron are mutually notably different, and the RSD values are extremely large for cyclohexane, and very large for benzene, diethylether, toluene, and DMSO (Tables 3 and 4). Such behaviour of the solvation enthalpies and Gibbs energies of the electron can be to some extent attributed to a well-known fact that radical anions, negatively charged species with one unpaired electron, are particularly challenging for computations. As expected, the results from the B2PLYP and B2PLYP-D3 methods are mutually consistent, but notably different from those from MP2. The B3LYP, B3LYP-D2, and B3LYP-D3 produced consistent results. Surprisingly, the Minnesota functionals often produced mutually inconsistent solvation enthalpy and Gibbs energy values of the electron. For example, $\Delta G_{\mathrm{sol}}\left(\mathrm{e}^{-}\right)$in cyclohexane vary from $-14.8 \mathrm{~kJ} \mathrm{~mol}^{-1}$ (M06-HF) up to $31.2 \mathrm{~kJ} \mathrm{~mol}^{-1}$ (M05-2X). Such behaviour of the Minnesota functionals has not been observed in our related work (Marković et al. 2016), and indicates their unreliable performance without the corresponding SMD solvation model. To put it simply, the performance of the Minnesota functionals is much better in combination with the SMD model, whereas other studied methods are only slightly dependent on solvation models.

Recall that both SMD (Marenich et al. 2009) and C-PCM (Cossi et al. 2003) are continuum solvation models, implying that they approximate the solvent by dielectric continuum that surrounds the solute molecules outside of a molecular cavity. SMD is based on the quantum mechanical charge density of a solute molecule interacting with a continuum description of the solvent. This model employs a single set of parameters (intrinsic atomic Coulomb radii and atomic surface tension coefficients) optimized over the M05-2X, B3LYP, and HF methods with different basis sets. On the other hand, C-PCM is a polarizable model in which the continuum is conductor-like. The conductor-like reaction field is due to an apparent polarization charge spread on the cavity surface.

In agreement with our previous findings, the least negative (most positive) values were produced by the MP2 method, followed by B2PLYP and B2PLYP-D3. The most negative (least positive) values were most often obtained by the M06-HF functional. The B3LYP, B3LYP-D2, and B3LYP-D3 also yielded notably low and mutually consistent $\Delta H_{\text {sol }}\left(\mathrm{e}^{-}\right)$and $\Delta G_{\text {sol }}\left(\mathrm{e}^{-}\right)$values.

As there are no pure experimental data on the solvation enthalpies or free energies of the proton and electron, it is difficult to conclude which method provides the values which match best with the exact values. A comparison of the results of the present investigation obtained by using C-PCM to those obtained by employing SMD reveals that C-PCM produces slightly more negative (less positive) solvation enthalpies and Gibbs energies of the electron, and less negative values for the proton. These results are also in accord with those suggested by 
(Rottmannová et al. 2013) and (Škorňa et al. 2014), where the IEF-PCM solvation model was used.

These data can be used to determine the enthalpies and Gibbs energies of the solvated proton and electron. Namely, the enthalpy $\Delta H\left(\mathrm{H}^{+}{ }_{\text {sol }}\right)$ and Gibbs energy $\Delta G\left(\mathrm{H}^{+}{ }_{\text {sol }}\right)$ of the solvated proton can be obtained by adding the $H\left(\mathrm{H}^{+}\right.$gas $)$and $G\left(\mathrm{H}^{+}\right.$gas $)$values to $\Delta H_{\text {sol }}\left(\mathrm{H}^{+}\right)$and $\Delta G_{\text {sol }}\left(\mathrm{H}^{+}\right)$, respectively. Similarly, the enthalpy $\Delta H\left(\mathrm{e}^{-}{ }_{\text {sol }}\right)$ and Gibbs energy $\Delta G\left(\mathrm{e}^{-}\right.$sol $)$of the solvated electron can be obtained by adding the $H\left(\mathrm{e}^{-}\right.$gas $)$and $G\left(\mathrm{e}^{-}\right.$gas $)$values to $\Delta H_{\text {sol }}\left(\mathrm{e}^{-}\right)$and $\Delta G_{\text {sol }}\left(\mathrm{e}^{-}\right)$, respectively. For this purpose the values proposed by Bartmess can be employed (Bartmess 1994).

\section{Conclusions}

Solvation enthalpies and Gibbs energies of the proton and electron are the quantities of significance in thermodynamic modeling of various natural and engineering processes. The enthalpies and Gibbs energies of the solvated proton and electron for a particular solvent can be issued from the corresponding solvation enthalpies and Gibbs energies. So far, the data on these quantities are still limited in the scientific literature. This work is an extension of our efforts aimed at providing the $\Delta H_{\text {sol }}\left(\mathrm{H}^{+}\right), \Delta G_{\text {sol }}\left(\mathrm{H}^{+}\right), \Delta H_{\text {sol }}\left(\mathrm{e}^{-}\right)$, and $\Delta G_{\text {sol }}\left(\mathrm{e}^{-}\right)$values for various solvents. In a recent study these quantities has been determined for twenty solvents of different polarities by means of eleven quantum mechanical methods in combination with the SMD solvation model (Marković et al. 2016). Exactly the same theoretical models in combination with the CPCM solvation were used in the present work.

It was found that different methods produce consistent values for the solvation enthalpy and Gibbs energy of the proton in all solvents, while the corresponding values for the electron are mutually notably different. Such behaviour of the solvation enthalpies and Gibbs energies of the electron can be to some extent attributed to a well-known fact that radical anions are particularly challenging for computations. As expected, the MP2, B2PLYP and B2PLYP-D3 methods showed extremely bad performance in reproducing $\Delta H_{\mathrm{sol}}\left(\mathrm{e}^{-}\right)$and $\Delta G_{\mathrm{sol}}\left(\mathrm{e}^{-}\right)$, whereas B3LYP, B3LYP-D2, and B3LYP-D3 yielded notably low, mutually consistent values which are comparable with the reported literature data. Surprisingly, the Minnesota functionals often produced inconsistent solvation enthalpy and Gibbs energy values of the electron. Such behaviour of these methods indicates their unreliable performance without the corresponding SMD solvation model, whereas other studied methods are only slightly dependent on solvation models.

A comparison of the results of the present investigation to those obtained by employing SMD reveals that C-PCM produces slightly more negative (less positive) solvation enthalpies and Gibbs energies of the electron, and less negative values for the proton, implying that the performance of C-PCM is similar to that of the IEF-PCM solvation model.

Acknowledgements This work was supported by the Ministry of Education, Science and Technological Development of Serbia, project No 172015 and 172016. 
Извод

\title{
Енталпија и Гибсова енергија солватације протона и електрона - утицај солватационих модела
}

\author{
J. Тошовић ${ }^{1 *}$, С. Марковић ${ }^{1}$, Д. Миленковић ${ }^{2}$, 3. Марковић $^{3}$ \\ ${ }^{1}$ Природно-математички факултет, Универзитет у Крагујевцу, 34000 Крагујевац, Србија \\ имејл: jelena.tosovic@kg.ac.rs \\ имејл: mark@kg.ac.rs \\ ${ }^{2}$ Истраживачко-развојни центар за биоинжењеринг БиоИРЦ, 34000 Крагујевац Србија \\ имејл: deki82@kg.ac.rs \\ ${ }^{3}$ Државни универзитет у Новом Пазару, 36300 Нови Пазар (Србија) \\ имејл: zmarkovic@np.ac.rs \\ *ллавни аутор
}

\section{Резиме}

Изведено је систематично испитивање енталпије и Гибсове енергије солватације протона и електрона у двадесет растварача различите поларности. Примењено је једанаест квантно механичких метода у комбинацији са базисним скупом 6-311++G(d,p) и солватационим моделом С-РСМ.

Установљено је да различите методе резултирају у конзистентним вредностима за енталпију и Гибсову енергију солватације протона код свих растварача, док се одговарајуће вредности за електрон међусобно доста разликују. Минесота функционали често воде до неконзистентних вредности за енталпију и Гибсову енергију солватације електрона, што указује на њихову непоузданост када се примењују без одговарајућег солватационог модела SMD. Остале испитане методе веома мало зависе од солватационог модела.

Поређење резултата овог истраживања са резултатаима који су добијени уз примену модела SMD показује да C-PCM производи нешто негативније (мање позитивне) енталпије и Гибсове енергије солватације електрона, док су вредности за протон мање негативне. Овакви резултати су у сагласности и са подацима из литературе који се односе на солватациони модел IEF-PCM.

Кључне речи: солватисани протон, солватисани електрон, двадесет растварача, солватациони модел С-РСМ

\section{References}

Atkins P W (1998). Physical Chemistry, sixth ed, Oxford University Press, Oxford.

Bartmess JE (1994). Thermodynamics of the electron and the proton, J. Phys. Chem., 98, 64206424.

Becke AD (1993). Density-functional thermochemistry. III. The role of exact exchange, J. Chem. Phys., 98, 5648-5652. 
Bockris JO, Reddy AKN (1998). Modern Electrochemistry, vol 1, Plenum, New York.

Coe JV (2001). Fundamental properties of bulk water from cluster ion data, Int. Rev. Phys. Chem., 20, 33-58.

Conway BE (1964). Proton solvation and proton transfer processes in solution, in: Bockris J O M, Conway B E (Eds), Modern Aspects of Electrochemistry, Butterworth, London.

Cossi M, Rega N, Scalmani G, Barone V (2003). Energies, structures, and electronic properties of molecules in solution with the C-PCM solvation model, J. Comp. Chem., 24, 669-681.

Curtiss LA, Raghavachari K, Redfern PC, Pople JA (2000). Assessment of Gaussian-3 and density functional theories for a larger experimental test set, J. Chem. Phys., 112, 73747383.

Donald WA, Demireva M, Leib RD, Aiken MJ, Williams ER (2010). Electron hydration and ion-electron pairs in water clusters containing trivalent metal ions, J. Am Chem. Soc., 132, 4633-4640.

Fifen JJ, Nsangou M, Dhaouadi Z, Motopon O, Jaidane N-E (2011). Solvent effects on the antioxidant activity of 3,4-dihydroxyphenylpyruvic acid: DFT and TD-DFT studies, Comp. Theor. Chem., 966, 232-243.

Fifen JJ, Nsangou M, Dhaouadi Z, Motapon O, Jaidane N-E (2013). Solvation energies of the proton in methanol, J. Chem. Theor. Comp., 9, 1173-1181.

Frisch MJ, Trucks GW, Schlegel HB, Scuseria GE, Robb MA, Cheeseman JR, Scalmani G, Barone V, Mennucci B, Petersson GA, Nakatsuji H, Caricato M, Li X, Hratchian HP, Izmaylov AF, Bloino J, Zheng G, Sonnenberg JL, Hada M, Ehara M, Toyota K, Fukuda R, Hasegawa J, Ishida M, Nakajima T, Honda Y, Kitao O, Nakai H, Vreven T, Montgomery J A Jr, Peralta JE, Ogliaro F, Bearpark M, Heyd JJ, Brothers E, Kudin KN, Staroverov VN, Kobayashi R, Normand J, Raghavachari K, Rendell A, Burant JC, Iyengar SS, Tomasi J, Cossi M, Rega N, Millam JM, Klene M, Knox JE, Cross JB, Bakken V, Adamo C, Jaramillo J, Gomperts R, Stratmann RE, Yazyev O, Austin AJ, Cammi R, Pomelli C, Ochterski JW, Martin RL, Morokuma K, Zakrzewski VG, Voth GA, Salvador P, Dannenberg JJ, Dapprich S, Daniels AD, Farkas Ö, Foresman JB, Ortiz JV, Cioslowski J, Fox DJ (2013). Gaussian 09, Rev D.1 Gaussian Inc, Wallingford CT.

Grimme S (2006). Semiempirical hybrid density functional with perturbative second-order correlation, J. Chem. Phys., 124, 034108-1-034108-16.

Grimme S (2006). Semiempirical GGA-type density functional constructed with a long-range dispersion correction, J. Comp. Chem., 27, 1787-1799.

Grimme S, Antony J, Ehrlich S, Krieg H (2010). A consistent and accurate ab initio parameterization of density functional dispersion correction (DFT-D) for the 94 elements H-Pu, J. Chem. Phys., 132, 154104-1-154104-19.

Halliwell HF, Nyburg SC (1963). Enthalpy of hydration of the proton, Trans. Faraday. Soc., 59, 1126-1140.

Han P, Bartels DM (1990). Reevaluation of Arrhenius parameters for $\mathrm{H} \bullet+\mathrm{OH}-\rightarrow(\mathrm{e}-)$ aq + H2O and the enthalpy and entropy of hydrated electrons, J. Phys. Chem., 94, 7294-7299.

Head-Gordon M, Pople JA, Frisch MJ (1988). MP2 energy evaluation by direct methods, Chem. Phys. Lett., 153, 503-506.

Marcus Y (1987). The thermodynamics of solvation of ions. Part 2. - The enthalpy of hydration at 298.15 K, J. Chem. Soc. Faraday Trans. 1, 83, 339-349.

Marenich AV, Cramer CJ, Truhlar DG (2009). Universal solvation model based on solute electron density and on a continuum model of the solvent defined by the bulk dielectric constant and atomic surface tensions, J. Phys. Chem. B, 113, 6378-6396.

Marković Z, Milenković D, Đorović J, Jeremić S (2013). Solvation enthalpies of the proton and electron in polar and non-polar solvents, J. Serb. Soc. Comp. Mech., 7, 1-9. 
Marković Z, Tošović J, Milenković D, Marković S (2016). Revisiting the solvation enthalpies and free energies of the proton and electron in various solvents, Comp. Theor. Chem., 1117.

Mejías JA, Lago S (2000). Calculation of the absolute hydration enthalpy and free energy of $\mathrm{H}+$ and $\mathrm{OH}-$, J. Chem. Phys., 113, 7306-7316.

Rashin AA, Namboodiri K (1987). A simple method for the calculation of hydration enthalpies of polar molecules with arbitrary shapes, J. Phys. Chem., 91, 6003-6012.

Rottmannová L, Škorňa P, Rimarčík J, Lukeš V, Klein E (2013). Solvation enthalpies of the proton in polar and non-polar solvents: Theoretical study, Acta Chim. Slovaca, 6, 60-63.

Schwarz HA (1991). Enthalpy and entropy of formation of the hydrated electron, J. Phys. Chem., 95, 6697-6701.

Shiraishi H, Sunaryo GR, Ishigure K (1994). Temperature dependence of equilibrium and rate constants of reactions inducing conversion between hydrated electron and atomic hydrogen, J. Phys. Chem., 98, 5164-5173.

Škorňa P, Rimarčík J, Klein E (2014). Solvation enthalpies of the electron in polar and nonpolar solvents: Theoretical study, Acta Chim. Slovaca, 7, 31-33.

Tao JM, Perdew JP, Staroverov VN, Scuseria GE (2003). Climbing the density functional ladder: Nonempirical meta-generalized gradient approximation designed for molecules and solids, Phys. Rev. Lett., 91, 146401-1-146401-4.

Tissandier MD, Cowen KA, Feng W Y, Gundlach E, Cohen MH, Earhart AD, Coe JV, Tuttle Jr TR (1998). The proton's absolute aqueous enthalpy and Gibbs free energy of solvation from cluster-ion solvation data, J. Phys. Chem. A, 102, 7787-7794.

Zhao Y, Schultz NE, Truhlar DG (2006). Design of density functionals by combining the method of constraint satisfaction with parametrization for thermochemistry, thermochemical kinetics, and noncovalent interactions, J. Chem. Theory and Comput., 2, 364-382.

Zhao Y, Truhlar DG (2008). Density functionals with broad applicability in chemistry, Acc. Chem. Res., 41, 157-167. 